\title{
High figure of merit (FOM) of Bragg modes in Au-coated nanodisk arrays for plasmonic sensing
}

\author{
Maxime Couture ${ }^{1}$, Thibault Brulé ${ }^{1}$, Stacey Laing ${ }^{2}$, Wenli Cui ${ }^{3}$, Mitradeep Sarkar ${ }^{4,5}$, \\ Benjamin Charron ${ }^{1}$, Karen Faulds ${ }^{2}$, Wei Peng ${ }^{3}$, Michael Canva ${ }^{4,5}$ and Jean-Francois \\ Masson ${ }^{1,6}$
}

${ }^{1}$ Département de chimie, Université de Montréal, CP. 6128 Succ. Centre-Ville, Montréal, Qc, Canada, $\mathrm{H} 3 \mathrm{C} 3 \mathrm{J7}$

${ }^{2}$ Bionanotechnologies, Department of Pure and Applied Chemistry, Technology Innovation Centre, University of Strathclyde, 99 George Street, Glasgow, G1 1RD, United Kingdom

${ }^{3}$ College of Physics and Optoelectronics Engineering, Dalian University of Technology, Dalian, 116024, China

${ }^{4}$ Laboratoire Charles Fabry Institut d'Optique Graduate School, Université Paris Sud, CNRS; 2 Avenue Augustin Fresnel, 91127 Palaiseau, France

${ }^{5}$ Laboratoire Nanotechnologies Nanosystèmes LN2 - CNRS, Université de Sherbrooke; Institut Interdisciplinaire d'Innovation Technologique, 3000 boul. de l'Université, Université de Sherbrooke, Sherbrooke, Qc, Canada, JIK 0A5

${ }^{6}$ Centre Québéeois sur les Matériaux Fonctionnels (CQMF)

*Corresponding author: jf.masson@umontreal.ca; tel: +1-514-343-7342

\begin{abstract}
We report that gold-coated nanodisk arrays of nearly micron periodicity have high figure of merit (FOM) and sensitivity necessary for plasmonic refractometric sensing, with the added benefit of suitability for surface-enhanced Raman scattering (SERS), large scale microfabrication using standard photolithographic techniques and a simple instrumental setup. Gold nanodisk arrays were covered with a gold layer to excite the Bragg modes (BM) which are the propagative surface plasmons localized by the diffraction from the disk array. This generated surface-guided modes, localized as standing waves, leading to highly confined fields confirmed by a mapping of the SERS

This is a peer-reviewed, accepted author manuscript of the following output : Couture, M., Brule, T., Laing, S., Cui, W., Sarkar, M., Charron, B., ... Masson, J-F. (2017). High figure of merit (FOM) of Bragg modes in Au-coated nanodisk arrays for plasmonic sensing. Small. (In press)
\end{abstract}


intensity and numerical simulations with 3D finite element method (3D FEM). The optimal gold-coated nanodisk arrays were applied for refractometric sensing in transmission spectroscopy with better performance than nanohole arrays and they were integrated to a 96-well plate reader for detection of IgY proteins in the $\mathrm{nM}$ range in PBS. The potential for sensing in biofluids was assessed with IgG detection in 1:1 diluted urine. The structure exhibits a high FOM of up to 46, exceeding the FOM of structures supporting surface plasmon polaritons (SPPs) and comparable to more complex nanostructures, demonstrating that sub-wavelength features are not necessary for high performance plasmonic sensing.

Keywords: Gold-coated nanodisk array, high FOM, multi-well plate reader, SERS, plasmonic sensing, Bragg mode

\section{Introduction}

The field of plasmonics has generated great interest in the past years due to a broad applicability for a series of important contemporary issues. ${ }^{1}$ Specifically, the usefulness of plasmonics has been shown for biomedical, environmental and food safety applications. $^{2-4}$ While the potential of plasmonic sensing has been shown for clinical sensing ${ }^{5}$, several issues remain to be addressed. For example, most sensors reported early on relied on a single measurement channel or low throughput. A significant number of applications require the measurement of multiple markers or reaching higher analytical throughput. Therefore, the field is rapidly moving towards higher multiplexing of plasmonic sensors ${ }^{6-9}$. To reach this goal, a high reproducibility must be achieved for a series of parameters, which include reproducible sensing performance of the device (plasmonic materials and fluidics), robust surface chemistry, repeatable immobilization of bioreceptors, optimization of the analytical conditions, and exquisite control on the external factors such as bulk properties of the sample and temperature among other factors. As one of the essential parameters to reaching the potential of plasmonic devices, the fabrication and characterization of reproducible nanostructures with high sensitivity are subject to intensive research activities ${ }^{10-12}$. 
Plasmonic substrates support surface plasmons (SP), which are coherent oscillations of free electrons at the interface of a metal and a dielectric. ${ }^{13}$ SPs are sensitive to refractive index variation, which allows a suite of plasmonic techniques to be used to detect binding events at the vicinity of the sensor's surface, hence the source of the general applicability and popularity of the technique to detect (bio)molecules. The coupling of light with SP in the classical technique of surface plasmon resonance (SPR) generates SPs that propagates on a thin gold film. ${ }^{1,3,14}$ Other coupling methods of light with SPs involve the use of nanoplasmonic structures such as 2D grating structures (in reflection or in transmission) or through resonances with colloidal nanoparticles leading to localized surface plasmon resonance (LSPR). ${ }^{1}$ 2D grating structures can be fabricated using several lithographic techniques ${ }^{15-17}$. While highly regular and reproducible, these fabrication techniques often necessitate infrastructure that can be costly to operate. However, they offer high sensitivity and currently constitutes a vibrant research field. Colloidal nanoplasmonics benefit from simple synthetic methods of the nanomaterials, which can then be self-assembled into more complex and functional architecture using self-assembly ${ }^{10,18}$. Colloidal nanostructures are also suited for the naked eye detection ${ }^{19}$, leading to simple design of sensors. The integration in lab-on-a-chip devices thus constitutes a significant advantage of 2D and colloidal nanoplasmonic materials ${ }^{20-22}$. Thereby, nanoplasmonic structures can solve current limitations of classical SPR, such as low-throughput capabilities for analyzing large numbers of samples and difficulty in miniaturization of equipment, ${ }^{23}$ and are highly sensitive for use in surface-enhanced spectroscopy such as SERS and metal enhanced fluorescence (MEF). ${ }^{24}$

The identification of nanoplasmonic structures exhibiting advantageous analytical properties is central to the development of the next generation of nanoplasmonic devices. Different nanostructures have been proposed to achieve highly sensitive and high figure of merit (FOM) plasmonic sensing. Colloidal nanostructures supporting LSPR have been widely popular for sensing due to recent advances in synthesis of metal colloids. ${ }^{25-27}$ These nanostructures are characterized with strong extinction in the visible to nearinfrared (NIR) spectral region, moderate sensitivity to bulk refractive index, but high sensitivity to molecular adsorbates in plasmonic sensing and in SERS. These structures 
are of different shapes and sizes such as nanorods, ${ }^{28}$ nanospheres, ${ }^{29}$ nanocubes, ${ }^{30}$ or nanostars, ${ }^{31}$ among others. Arrays of individual nanostructures have also been fabricated on solid substrates with properties similar to colloids in suspension, including arrays of nanotriangles, ${ }^{32}$ nanorings ${ }^{33}$, nanocrescents ${ }^{34}$, or nanodisks ${ }^{35}$ among others. Since the first report on nanodisks ${ }^{36}$, they have been increasingly used for various applications in plasmonics ${ }^{37-41}$. Alternatively, there has also been a lot of interest in developing nanostructures that support propagating SPs. These nanostructures typically have higher bulk refractive index sensitivity than LSPR, higher FOM (FOM = bulk RI sensitivity / full width at half-maximum (FWHM)) and achieve better refractive index resolution. ${ }^{42}$ For example, nanoslits ${ }^{43}$, nanovoids ${ }^{44}$, nanohole arrays ${ }^{45}$, and nanogratings ${ }^{46}$ have been reported for plasmonic sensing. Despite all these significant advances in nanostructure design, it remains one of the contemporary challenges to develop a reproducible nanostructure with high sensitivity, high FOM and broad applicability in a series of plasmonic techniques.

To address these challenges, we studied gold-coated nanodisk arrays and report the generation of sharp resonances in transmission spectroscopy measurements in the near-infrared (NIR) region of the spectrum ideal for biosensing applications. In periodic structures with an underlying metallic film, the Bragg Modes (BMs) are excited due to the change of the in-plane wavevector of the PSP $\left(k_{P S P}\right)$ owing to the periodicity of the array. For this work, we selected a nanodisk array structure with a large periodicity (1200 $\mathrm{nm})$ and disk diameter $(720 \mathrm{~nm})$ where resonant coupling of the localized and propagative plasmons are not expected to occur for the wavelengths used in this work. Advantages of working with larger structural parameters include tuning of the resonance in the so-called biological window in the NIR and the possibility to fabricate the arrays by conventional photolithography. Microfabrication on a large scale with high surface uniformity is highly desirable for integration of plasmonic structures in devices. While most plasmonic structures reported excel in few of the analytical parameters of importance for sensing using refractometric (such as sensitivity, FWHM, FOM, RI resolution, $\mathrm{S} / \mathrm{N}$, and reproducibility among others) and surface-enhanced spectroscopies, 
we report excellent analytical properties of the gold-coated nanodisk arrays in this series of important parameters.

\section{Results and Discussion}

Gold nanodisk arrays are usually fabricated by electron beam lithography (EBL) and exhibit sub-wavelength features. ${ }^{47,48}$ The fabrication of gold-coated nanodisk arrays was based on the same approach we used in a recent paper to manufacture nanohole arrays of the same periodicity (Figure 1). ${ }^{49}$ In brief (detailed experimental procedures are provided below), the microfabrication of nanodisk arrays was achieved by exposing UV light through a chromium mask on a positive photoresist, which was spin-coated on a 4" glass wafer. The mask consisted of an array of chromium disks with a periodicity of 1200 $\mathrm{nm}$ and a diameter of $720 \mathrm{~nm}$. After the exposition step, the wafer was developed and a nanohole arrays pattern was produced on the glass wafer. Thereafter, the wafer was metalized with a layer of chromium (1 nm) and a layer of gold (10 to $75 \mathrm{~nm})$ was deposited to yield a nanodisk array on the wafer. Then, the photoresist was lifted off the wafer to display the array of gold nanodisks. The last step of the process consisted of coating another layer of gold (10 to $75 \mathrm{~nm}$ ) onto the nanodisk arrays. The gold-coated nanodisk arrays were named for their nanodisk height (NDXX, where XX is the height in $\mathrm{nm}$ ) and their gold film thickness (filmYY, where YY is the thickness in nm), thus a substrate with disks of $75 \mathrm{~nm}$ and a film of $75 \mathrm{~nm}$ is termed ND75film75. This fabrication technique generated gold-coated nanodisk arrays on a large area (SEM image provided in Figure 1), with great speed and high surface uniformity ( $<10 \%$ variation).

We first studied the optical properties of the nanostructures. The localized plasmon resonance of the isolated disks was measured at $1.6 \mu \mathrm{m}$ for free nanodisks. When the array was coated with a gold layer, the plasmon resonance of the disk was expected to shift, but not significantly. Thus, we did not expect any localized surface plasmon resonance of the gold-coated nanodisk arrays in the working wavelengths (Visible-NIR) used for this article. Thus, we did not consider any further coupling possibilities of the PSP and the localized plasmon resonances of the disks. ${ }^{50,51}$ 
The $75 \mathrm{~nm}$ layer of gold below the nanodisk arrays (disk height of $75 \mathrm{~nm}$ ) led to a series of plasmonic bands associated to BM in the visible-NIR region (Figure 2). The plasmon resonances were excited when the wavevector of the PSP matched the wavevector of the diffracted orders from the array. The equation for the condition of exciting the diffracted orders $(i, j)$ is given in terms of the excitation wavelength $(\lambda)$, dielectric constants $(\varepsilon)$ of the metal $(m)$ and solution $(d)$, the refractive index of the glass support $\left(\eta_{D}\right)$ and incidence angle $(\theta)$ as

$$
\begin{aligned}
& \lambda=\frac{\mathrm{P}}{\sqrt{\frac{4}{3}\left(\mathrm{i}^{2}+\mathrm{ij}+\mathrm{j}^{2}\right)}}\left(\sqrt{\frac{\varepsilon_{\mathrm{m}} \varepsilon_{\mathrm{d}}}{\varepsilon_{\mathrm{m}}+\varepsilon_{\mathrm{d}}}}-\eta_{\mathrm{D}} \sin \theta\right) \\
& \lambda=\frac{\mathrm{P}}{\sqrt{\frac{4}{3}\left(\mathrm{i}^{2}+\mathrm{ij}+\mathrm{j}^{2}\right)}}\left(\sqrt{\frac{\varepsilon_{\mathrm{m} \varepsilon_{\mathrm{d}}}}{\varepsilon_{\mathrm{m}}+\varepsilon_{\mathrm{d}}}}-\eta_{\mathrm{D}} \sin \theta(\mathrm{i} \cos (\phi)+\mathrm{j} \cos (\phi+60)+\mathrm{i} \sin (\phi)+j \sin (\phi+60))\right)
\end{aligned}
$$

These plasmonic modes highly depend on the periodicity $(P)$ of the array rather than the nanodisk shape. ${ }^{52}$ Equation 1 is valid only for hexagonal arrays of nanostructures such as used in this article and considered a rotation angle of $0^{\circ}$ (Figure 1) ${ }^{49}$, while equation 2 considers all rotation angles $(\phi)$, also for hexagonal arrays. Analytical calculations using equation 1 were then carried out to estimate the BM coupling conditions and to determine the position of the different orders of BM (Figure 2). The predicted resonances calculated with equation 1 were in good agreement with the experimental positions of the BM for different orders (Figure 2A). For example, the experimental resonance wavelength of $641 \mathrm{~nm}$ was also in good agreement with the 3D FEM prediction at $644 \mathrm{~nm}$. Based on equation 1, the plasmonic resonances observed in air for the incidence angles of $0^{\circ}$ and $5^{\circ}$ were assigned to the $(1,1)$ and $(2,-1)$ modes (shifting to shorter wavelengths) and the $(-1,-1)$ and $(-2,1)$ modes (shifting to longer wavelengths) (Figure 1). We must mention here that the current experiments were not performed with polarized light, which is common for sensing applications of plasmonic nanostructures. However, polarization has been previously shown to influence the spectra of disordered nanohole arrays ${ }^{53}$. In that case, p-polarization showed angular dependence, which was not observed for s-polarization due to the electric field orientation in the plane 
of the nanostructure for the latter case. These results showed that a large number of BM can be excited with the gold-coated nanodisk arrays and the impact of the excitation conditions on these resonances.

The sample was then rotated $90^{\circ}$ to demonstrate the effect of rotation angle on the excitation of the BM and the difference in the plasmon dispersion curves (equation 2 and Figure 2B). At a rotation angle of $90^{\circ}$, several bands such as the $(1,0)$ and $(-1,0)$ bands are orthogonal to the plane of incidence, which has a consequence of invariability of the plasmon resonance wavelength (excited at nearly $1.1 \mu \mathrm{m}$ ) in relation to the excitation angle, an effect that was also seen for the $(-1,2)$ and $(1,-2)$ bands at a rotation angle of $0^{\circ}$. Consequently, the plasmon dispersion curves at a rotation angle of $90^{\circ}$ had fewer bands than at a rotation angle of $0^{\circ}$.

We then measured the plasmonic properties of 16 gold-coated nanodisk arrays with various disk heights and film thicknesses to evaluate the optimal excitation conditions of the BM. These different plasmonic structures were studied in transmission for a range of incident angles of $0^{\circ}$ to $30^{\circ}$ and all plasmonic dispersion curves are reported in figure S1. Equation 1 accurately predicted $( \pm 12 \mathrm{~nm})$ the resonances observed at an incidence angle of $0^{\circ}$ for the gold-coated nanodisk arrays of different heights and film thicknesses. This observation further supported the excitation of BM for the goldcoated nanodisk arrays of the different nanodisk heights and film thicknesses. In addition, the resonance of the gold-coated nanodisk arrays was invariant $( \pm 5.5 \mathrm{~nm})$ with the height of the nanodisk (10 to $75 \mathrm{~nm}$ ). If a localized plasmon was excited in the NIR spectral range, the plasmon resonance would have strongly depended on the aspect ratio of the disk, as previously reported ${ }^{54}$, which was not the case for the gold-coated nanodisk arrays. However, the plasmon resonance slightly increased from 631 to $644 \mathrm{~nm}$ for thicker $\mathrm{Au}$ films (from 25 to $75 \mathrm{~nm}$ ), which is also in agreement with a redshift observed for thicker Au films in classical propagative SPR measurement in the Kretschmann configuration. This observation is valid for symmetric modes, but it is important to mention that other modes can exist in finite films. We also observed that nanodisks of heights of $10 \mathrm{~nm}$ were insufficient to efficiently excite the BM, excepted with a film 
thickness of $75 \mathrm{~nm}$ for which a very weak resonance was observed at $640 \mathrm{~nm}$, a resonance wavelength in agreement with other disk heights. Nanodisks of small heights failed to effectively diffract the surface plasmon and thus, light cannot couple to the propagating plasmon. Film heights on the order of 10 to $20 \mathrm{~nm}$ do not typically support propagating surface plasmons in classical SPR experiments using smooth films and the Kretschmann configuration of SPR. In agreement, we generally did not observe the excitation of the propagating surface plasmon of gold-coated nanodisk arrays with $10 \mathrm{~nm}$ Au films, with the exception of nanodisks of $50 \mathrm{~nm}$, for which, a weak resonance was observed. Overall, these results demonstrated that sufficient thicknesses (50 and $75 \mathrm{~nm}$ ) of the gold film and nanodisks are the necessary conditions to strongly supports a propagative surface plasmon and excite the BMs for gold-coated nanodisk arrays.

The sensitivity of the gold-coated nanodisk arrays was then evaluated for sensing purposes. The excitation of these nanostructures at normal incidence in aqueous solutions shifted the BM, which was at nearly $640 \mathrm{~nm}$ (normal incidence) in air, towards wavelengths of nearly $840 \mathrm{~nm}$. The bulk refractive index sensitivity ( $\sim 600 \mathrm{~nm} / \mathrm{RIU})$, and RI resolution ( $1.8 \times 10^{-5}$ RIU) were relatively similar for every structure (Table 1$)$, which was expected since the refractive index sensitivity (RIS) of a plasmonic grating nanostructure varies mainly from the variation of the periodicity..$^{55}$ The refractive index resolution was measured from the noise level measured from a temporal series of spectra. The RI resolution obtained with the gold-coated nanodisk arrays was identical to a cavity-coupled plasmonic device reported recently ${ }^{42}$ and of nanodisk arrays (estimated from data in ref. ${ }^{56}$ ), and was better than RI resolution of nearly $10^{-4}$ RIU typically reported for the classical spherical Au nanoparticle ${ }^{57}$. If optical detection methods other than transmission are used (phase, microscopy, optical fibers), refractive index resolution of $10^{-6}$ RIU or better can be achieved ${ }^{58}$ but oftentimes with added complexity of the instrumental setup. Furthermore, the plasmonic signal of the BM in water was very sharp with a full width at half maximum (FWHM) of $14 \mathrm{~nm}$ for the gold-coated nanodisk arrays. In comparison, nanodisk arrays had a smaller RI sensitivity than nanohole arrays of the same periodicity ( $900 \mathrm{~nm} / \mathrm{RIU}$ ) but their FWHM was 5 -fold smaller in comparison to the same nanohole arrays. ${ }^{49}$ The sensitivity and FWHM of gold-coated nanodisk arrays 
were close to the best values reported for other plasmonic nanostructures. In brief, the sensitivity is typically less than $400 \mathrm{~nm} /$ RIU for LSPR nanostructures, and while it varies with the periodicity for arrays of nanostructures, it is generally comprised between 200 and $700 \mathrm{~nm} / \mathrm{RIU}$ for arrayed nanostructures with resonances below $1000 \mathrm{~nm} .{ }^{42} \mathrm{In}$ addition, the FWHM is typically on the order of a few tens to hundreds of nanometers for LSPR and it was reported within the range of 4 to $36 \mathrm{~nm}$ for SPP. ${ }^{42}$ Our reported value for the FWHM of $14 \mathrm{~nm}$ for gold-coated nanodisk arrays was also close to the best nanostructures. The narrow linewidth was due to the excitation of the Bragg mode of the nanostructure array, which are typically narrower than propagating plasmons (PSP) on smooth films. The area of perfectly ordered nanostructure also contribute to narrowing the linewidth and achieving small FWHM.

The FOM (FOM = RIS/FWHM; calculated in using values in $\mathrm{nm}$ ) in plasmonic structure is a quantitative parameter providing an indication of the resolution in refractometric sensing. High FOM structures generally have better resolution and thus, lower noise and ultimately leading to sensors with better limits of detection. It is thus customary to compare the performance of plasmonic structures using FOM. From this data, we obtained a FOM that varied from 42 to 46 for gold-coated nanodisk arrays, which once again fared as one of the best overall plasmonic nanostructures reported to date. For example, structures supporting a LSPR resonance such as nanoparticle arrays $(\mathrm{FOM}=25)^{59}$, nanobar arrays $(\mathrm{FOM}=4.5)^{60}$, nanodisk $(\mathrm{FOM}=29){ }^{61}$, Au octamers $(\mathrm{FOM}=5.7)^{62}$, or a "XI" nanostructure $(\mathrm{FOM}=4.7)$ generally had FOM from 0.6 to 29 , while if a Wood's anomaly was excited, the FOM increased to $38^{63}$. Structures supporting a SPP are generally on the order of FOM $=23$ to 39, which includes the FOM of a thin Au film classically used in SPR sensing (FOM $=25$ to 39) ${ }^{64}$. Significantly higher FOM can be achieved with SPP with Fano resonances, where the FOM ranges from $39^{65}$ and $252^{43}$, and often exploits subwavelength nanostructures such as the nanoslits structures ${ }^{43,66}$, grating structures ${ }^{67}$, suspended nanohole arrays ${ }^{68}$ or nanomushrooms ${ }^{69}$ to reach higher FOM. A more extensive list of FOM for a series of nanostructures was provided elsewhere for comparison purposes. ${ }^{42}$ However, one must 
keep in mind that the FOM of a plasmonic structure is not an absolute measurement of the sensing performance and low FOM plasmonic structures have been shown to perform well in sensing. ${ }^{70}$ Hence, the gold-coated nanodisk arrays are well positioned due to their combination of high FOM, sensitivity and RI resolution to achieve highly sensitive refractometric measurements. The relatively simple photolithographic fabrication is also an advantage of the gold-coated nanodisk arrays.

While some nanostructures may have greater performance for one of the parameters mentioned in the previous paragraph, it is rare to find a nanostructure excelling in all these analytical parameters, especially with micron size features. For this purpose, we compared the performance of gold-coated nanodisks arrays with nanohole arrays, nanodisk arrays and crossed nanogratings, which are nanostructures bearing structural similarities with gold-coated nanodisk arrays (Table 2). While a list provided here cannot be exhaustive, readers are referred to Homola and coworkers ${ }^{58}$ and Martin and coworkers ${ }^{42}$, which provided recently comprehensive overviews of the analytical parameters for nanoplasmonic structures. Crossed gratings had similar performances to gold-coated nanodisk arrays with the exception of a lower FOM of 14, nanodisk arrays and nanohole arrays consistently fared worse or equally to gold-coated nanodisk arrays depending on the parameter compared. We then compared our results to two plasmonic substrate with exceptional FOM. Nanomushrooms had superior sensitivity, FWHM and FOM, but had a RI resolution 10x worse than the gold-coated nanodisk arrays. SERS performance for this nanostructure has not been reported. Suspended nanohole arrays have a FOM of 162 and excellent sensitivity and FWHM, but are expected to have lower SERS EF (close to conventional nanohole arrays). They are relatively complex to fabricate and are suspected to be more fragile due to the suspended thin film. One clear distinction between these nanostructures and the gold-coated nanodisk arrays is that all nanostructures had features smaller than $300 \mathrm{~nm}$, with a periodicity smaller than $600 \mathrm{~nm}$. The gold-coated nanodisk arrays are significantly larger with features of $720 \mathrm{~nm}$ and $1200 \mathrm{~nm}$ periodicity, which shows that sub-wavelength features are not necessary for achieving high analytical performance. 
One of the main parameters dictating the performance of a plasmonic sensor for biosensing purposes is the distribution of the EM field. ${ }^{4}$ The surface sensitivity of a sensor characterizes the interaction of the plasmonic field at the metal/air or metal/solution interface for the detection of biological recognition events. Surface sensitivity of the gold-coated nanodisk arrays was assessed with a biosensor for IgY in PBS. For this test, a custom multi-well plate reader was used in order to achieve a broadly multiplexed platform. The multi-well plate reader allowed monitoring of up to 96 wells (volume of $100 \mu \mathrm{L} /$ well) in a single experiment and was used without modification from a previous report. ${ }^{49}$ The biosensor was constructed to include a peptide monolayer 71,72 for minimizing nonspecific adsorption of biofluids, on which a polyclonal anti-IgY was immobilized with standard EDC-NHS chemistry as previously reported. ${ }^{73,74}$ The sensor was then subjected to direct detection of $100 \mathrm{nM}$ of $\operatorname{IgY}$ in triplicate measurements (in different wells of the 96-well plate reader), followed by a secondary detection using the same polyclonal anti-IgY antibody. A control in absence of IgY was performed with a triplicate of wells to validate the specificity of the secondary detection step. We obtained a plasmonic shift of $0.1 \pm 0.1 \mathrm{~nm}$ in absence of $\mathrm{IgY}$, confirming the specificity of the secondary detection step. From these data, we estimated the surface sensitivity at the limit of detection to be approximately $5 \mathrm{pg} / \mathrm{mm}^{2}$. Differences in gold film and nanodisk thicknesses did not significantly affect the surface sensitivity of the sensors, but the goldcoated nanodisk array with ND75film75 exhibited the best overall performance (Table 1) and was thus used for the following experiments. We then measured the surface sensitivity of the gold-coated nanodisk arrays with a calibration curve of IgY (Figure 3 ). The noise level of gold-coated nanodisk arrays was lower than for nanohole arrays. Both calibration curves were nearly identical, but the plasmonic response was slightly better for nanohole arrays at higher concentration (>100 nM).

Biosensing in complex matrices is one of the main challenges of plasmonic sensing because of the nonspecific adsorption of background proteins on refractometric sensors. ${ }^{1,71}$ To demonstrate the potential of this sensing platform for more realistic sample measurements, biosensing of human $\mathrm{IgG}$ was performed in human urine. IgG detection in urine can serve for detection of urinary tract infections among other 
pathologies. The sensor was modified with anti-human $\operatorname{IgG}$ to capture $\operatorname{IgG}$ in the sample. Since human urine contains IgG at concentrations on the order of a few $\mu \mathrm{g} / \mathrm{mL}$ (about a few tens of nanomolar) ${ }^{75}$, we performed a standard addition assay where human urine was spiked with an additional concentration of 100 and $1000 \mathrm{nM}$ of human IgG. Once again, a control consisting of injecting human urine without further spiking with human IgG led to a plasmonic shift 3-fold smaller $(\Delta \lambda=0.15 \pm 0.02 \mathrm{~nm})$ than for the detection of a human urine sample supplemented with $100 \mathrm{nM}$ human $\operatorname{IgG}(\Delta \lambda=0.39 \pm 0.02 \mathrm{~nm})$. The shift of the sensor further increased with the addition of $1000 \mathrm{nM} \mathrm{IgG} \mathrm{in} \mathrm{human}$ urine $(\Delta \lambda=0.52 \pm 0.03 \mathrm{~nm})$. This indicated that the sensor was able to differentiate human IgG from background noises from analytes in a complex matrix.

To further characterize the gold-coated nanodisk arrays and to evaluate the potential of this platform in surface-enhanced spectroscopy, we have studied the properties of this plasmonic substrate in SERS and numerically simulated the field distribution on the nanostructure. Typical nanodisk arrays (without the gold film) have been extensively reported in SERS applications with enhancement factors (EF) of $10^{6}$ $10^{7} .{ }^{76-78}$ The high localization of the plasmonic field near nanodisks allowed a strong enhancement of the EM field intensity. The optimal conditions will depend on the geometrical parameter of the nanodisk, as the EM enhancement is wavelength-dependent and requires coupling of the wavelength of the laser with the plasmonic resonance of the nanostructure. ${ }^{24,79}$ For our gold-coated nanodisk arrays, the plasmon resonance was excited at wavelengths in air $(\lambda=641 \mathrm{~nm})$ and water $(\lambda=840 \mathrm{~nm})$ close to the range of wavelengths from common Raman lasers of 633 and $785 \mathrm{~nm}$.

A false color Raman map of gold nanodisk arrays (ND75film75) was generated to assess the localization of the plasmonic field (Figure 4) using the intensity of the peak at $1335 \mathrm{~cm}^{-1}$ from the spectrum of nitrobenzenethiol (4-NBT). The Raman map revealed that the highest intensity of Raman scattering was located near the edge of the nanodisks. These results correlated with the previously reported theory that the plasmonic field of the BM should be highly confined at the edges of the nanodisk. ${ }^{51}$ Slight differences were observed in the experimental and 3D FEM data (Figure 4). The Raman maps showed a 
relatively even field around the circumference of the nanodisks, while the 3D FEM simulations reported a dipolar mode of the field. This difference is likely due to the conical irradiation of the substrate due to the numerical aperture of the objective.

The Raman map could not distinguish if the highest EM field was located at the bottom or top of the nanodisk. Therefore, we simulated the field distribution using 3D FEM, where in these simulations the field impinged at the metal-air interface, as for the Raman experiments. The Raman map was in good agreement with the 3D FEM data of the electromagnetic field distribution in the X-Y plane for the same gold-coated nanodisk arrays (Figure 4). Indeed, the plasmonic field at a wavelength of $644 \mathrm{~nm}$ described a donut shape on the edges of the nanodisks for $\mathrm{Z}$ values of $75 \mathrm{~nm}$ (gold film/nanodisk interface) and $150 \mathrm{~nm}$ (top of nanodisk). These results demonstrated that the field intensity at the edges of the disk was higher at the interface of the nanodisk and air ( $\mathrm{Z}=$ $150 \mathrm{~nm}$ ). In addition, the simulations showed the plasmon field leaking in the gold film and creating interference patterns around the nanodisk $(Z=75 \mathrm{~nm})$. The 3D field simulations demonstrated the high confinement of the field distribution near the top of the nanodisk, a location in agreement with the Raman images. The field distribution was also similar to 3D FEM simulations for transmission measurements, such as the ones reported for the plasmonic sensing experiments above.

Enhancement factors (EF) are often compared to evaluate the potential of plasmonic structures in SERS. Gold-coated nanodisk arrays led to an estimated EF of $10^{7}$, which is slightly higher than that reported by Gillibert et al. for a similar structure ${ }^{52}$ and slightly lower than the SERS enhancement of $10^{8}$ recently reported on a nanotriangle arrays on a gold film. ${ }^{80}$ SERS EF on the order of $10^{7}$ to $10^{8}$ were reported to be sufficient for single molecule studies ${ }^{81}$ and typical SERS EF reported in the literature ${ }^{82}$ ranges from $10^{5}$ to $10^{7}$, such as for cavity nanohole arrays ${ }^{83}$, but can exceed this value in aggregated nanoparticles. Higher SERS EF could be achieved using nanodisk arrays with smaller features, as reported elsewhere using nanoimprint lithography ${ }^{84}$. The SERS response from different locations was highly reproducible with about $7 \%$ of coefficient of variation measured on 576 spots on the gold-coated nanodisk arrays, which was also 
evident from the relative homogeneity of the SERS intensity on the Raman map (Figure 4). The periodic structure and the lower EF can explain the better SERS reproducibility of this plasmonic substrate. Thus, gold-coated nanodisk arrays can also serve as effective SERS substrates.

\section{Conclusions}

In conclusion, we developed gold-coated nanodisk arrays with great potential for biosensing applications. A screening of the plasmonic properties of 16 structures demonstrated that the plasmonic properties were superior for thicker gold film and nanodisk heights ( $\geq 50 \mathrm{~nm}$ ). The refractive index sensitivity (about $600 \mathrm{~nm} / \mathrm{RIU}$ ), resolution (1.8 x 10 $0^{-5} \mathrm{RIU}$ ), and FOM (up to 46) were superior to most plasmonic substrates reported to date. While higher refractive index, higher FOM or SERS enhancement can be achieved in a few plasmonic nanostructures, the previously reported nanostructures required features with significantly smaller dimensions and often excelled in one of these parameters. The gold-coated nanodisk arrays performed well in all these analytical parameters, were fabricated using simpler techniques and also showed good surface sensitivity towards protein detection. We also demonstrated that gold-coated nanodisk arrays can be easily integrated with a 96-well plate reader and we confirmed their great potential for high throughput label-free biosensing. Finally, a mapping of the SERS intensity and 3D FEM simulations revealed that the plasmonic field of the BM was highly confined at the edges of the nanodisk. This enhanced EM field generated a high SERS EF of approximately $10^{7}$. Thus, gold-coated nanodisk arrays showed great potential for plasmonic applications.

\section{Methods}

\section{Microfabrication}

Gold-coated nanodisk arrays and nanohole arrays were manufactured by photolithography similarly to our previous publications. ${ }^{49,85}$ The fabrication of goldcoated nanodisk arrays was achieved with a positive photoresist (OIR 674-11) and a 
sacrificial layer (LOR1A) using the same patterned mask as for nanohole arrays. A layer of $\mathrm{Cr}(1 \mathrm{~nm})$ and different thicknesses of $\mathrm{Au}(10,25,50$ or $75 \mathrm{~nm})$ were evaporated on the patterned wafer using electron beam deposition. Resins were lifted off from the wafer by sonication in remover PG. This resulted in a wafer covered with gold nanodisk arrays where the deposited thickness of gold corresponded to the height of the disk. The periodicity and diameter of the nanodisk arrays were respectively $1200 \pm 20$ and $650 \pm 20$ $\mathrm{nm}$. Then, another layer of gold was evaporated on the gold nanodisk arrays for different gold thicknesses (10, 25, 50 or $75 \mathrm{~nm}$ ). A total of 16 gold-coated nanodisk arrays were manufactured using this methodology. The structures were identified from the height of the nanodisk (ND75 for a $75 \mathrm{~nm}$ high nanodisk) and gold film (film75 for a $75 \mathrm{~nm}$ thick gold film) evaporation steps. Hence, a structure labeled ND75film75 had a nanodisk arrays of $75 \mathrm{~nm}$ thick followed by a deposition of $75 \mathrm{~nm}$ of gold covering the nanodisk arrays. The structures of the nanohole arrays and nanodisk arrays were confirmed by scanning electron microscopy (SEM, Jeol). The periodicity and diameter of the nanodisk were measured by atomic force microscopy (AFM, Witec).

\section{Optical measurements and plasmonic sensing}

Gold-coated nanodisk arrays were excited in transmission spectroscopy using a homemade fluidic cell and a halogen lamp. The plasmon dispersion curves were obtained by measuring the plasmonic signal in air for each gold-coated nanodisks arrays at various incident angles from $0^{\circ}$ to $30^{\circ}$ using a manual goniometer. Spectra were collected with a PI-Acton spectrophotometer equipped with a grating of $150 \mathrm{~g} \mathrm{~mm}^{-1}$ (set from 415 to 975 $\mathrm{nm}$ ) and a CCD camera (Andor model DU401A-BR-DD-152). The transmission signal of the corresponding gold film thickness was used as a reference for the normalization of the plasmonic signal for each gold-coated nanodisk arrays. The bulk refractive index sensitivity of the sensors was determined with aqueous sucrose solutions (1.33-1.36 RIU) using a fluidic cell. A screening of the surface sensitivity of several nanodisks arrays was achieved with a custom multi-well plate reader and 96-well plate as described elsewhere ${ }^{49}$. For these measurements, a grating of $600 \mathrm{~g} \mathrm{~mm}^{-1}$ was used and set from 785 to $914 \mathrm{~nm}$. Direct and secondary detection of IgY proteins (Cedarlane) using anti-IgY antibodies (Cedarlane) was achieved with the plasmonic reader for several structures 
based on previously reported methods. ${ }^{73,74}$ Each sensor was measured in triplicate and a control was achieved to assess nonspecific adsorption. A calibration curve for detection of IgY in PBS (10 to $1000 \mathrm{nM}$ ) was performed for gold-coated nanodisk arrays and compared to nanohole arrays using the fluidic cell setup. Detection of human $\mathrm{IgG}$ protein (100 nM, $1000 \mathrm{nM}$ and control) was also done in PBS and diluted urine (1:1 in PBS:urine) with the fluidic cell for the nanodisk arrays. Furthermore, the LSPR signal of the nanodisk arrays and gold-coated nanodisk arrays (ND75film75) was measured with a Cary 6000i UV-vis-NIR spectrophotometer set from 500 to $1800 \mathrm{~nm}$.

\section{Raman spectroscopy measurements}

SERS enhancement factors (EF) of gold-coated nanodisk arrays (ND75film75) was estimated using a confocal Raman microscope (Renishaw inVia) with a grating of $600 \mathrm{~g} \mathrm{~mm}^{-1}$, a $633 \mathrm{~nm}$ laser excitation with $4.4 \mathrm{~mW}$ at the sample and a 50x/0.75 objective. Samples were functionalized overnight in a $1 \mu \mathrm{M}$ solution of 4nitrobenzenethiol (4-NBT). Raman spectra were acquired at a frequency of $1 \mathrm{~Hz}$ for a total of 20 spectra average per acquisition. A Raman image of the nanodisk array (ND75film75) was collected on a WITec alpha 300 with a $633 \mathrm{~nm}$ laser excitation wavelength using the piezo-driven XYZ scan stage and a grating of $600 \mathrm{~g} \mathrm{~mm}^{-1}$ coupled to a CCD camera. The spectra were collected with an integration time of 0.1 seconds using a $100 \mathrm{x} / 0.9$ objective with a laser power of around $1 \mathrm{~mW}$ at the sample. Image scans of $10 \mu \mathrm{m}$ x $10 \mu \mathrm{m}$ were obtained using a $100 \mathrm{~nm}$ step size. WITec Project software was used to background subtract the spectra using a first order polynomial fit and false color images were generated based on the intensity of the 4-NBT peak at $1335 \mathrm{~cm}^{-1}$.

The SERS EF of the ND75film75 structure was determined using equation 2 where ISERS and IRaman correspond respectively to the intensity of the 4-NBT band at 1335 $\mathrm{cm}^{-1}$ for SERS and normal Raman. ${ }^{24}$

$$
E F=\left(\frac{\frac{I_{S E R S}}{N_{S E R S}}}{\frac{I_{\text {Raman }}}{N_{\text {Raman }}}}\right)
$$

where $\mathrm{N}_{\text {SERS }}$ and $\mathrm{N}_{\text {Raman }}$ are respectively the number of excited molecules generating the SERS and Raman signal. The value of N SERS was based on the surface coverage of 4-NBT around a nanodisk. This value was determined based on the coverage 
ratio given in Saverot et al. $\left(0.54 \mathrm{~nm}^{2}\right)^{86}$ and the estimation of the active area of high intensity EM field was approximately $12,000 \mathrm{~nm}^{2}$ based on figure 5 . N $\mathrm{N}_{\text {SERS }}$ was thus approximately 6,400 molecules. The value of $\mathrm{N}_{\text {Raman }}$ was determined based on the Raman scattering from a solution of 4-NBT where the number of 4-NBT molecules present in the solution within the laser interrogation volume was estimated to be approximately $4 \times 10^{7}$ molecules. ISERS and $I_{\text {Raman }}$ were respectively 3,984 counts/s and 3.3 counts/s.

\section{Simulation}

The theoretical dispersion curves of the BM were calculated using a Matlab algorithm based on equation 1 . The values were determined for an array having a periodicity of $1200 \mathrm{~nm}$ and for incident angles of $0^{\circ}$ to $30^{\circ}$. 3D finite element method (3D FEM) simulations were also carried for the gold-coated nanodisk arrays (ND75film75) in air using COMSOL multiphysics software package for the wavelength region of 600 to $1000 \mathrm{~nm}$. All spectra were normalized with the incident light field. The normalized electromagnetic field distribution in the $\mathrm{X}-\mathrm{Y}$ plane was plotted at the peak wavelength under polarized normal incidence. The region from $Z=0,75$, and $150 \mathrm{~nm}$ corresponded to the BK7 glass substrate $(Z=0 \mathrm{~nm})$, interface of the gold film $(Z=75$ $\mathrm{nm})$ and top of the nanodisks $(Z=150 \mathrm{~nm})$.

\section{Acknowledgements}

The authors thank financial support from the National Science and Engineering Research Council (NSERC) of Canada (MC and JFM), of the Canada Foundation for Innovation (JFM), the Leverhulme Trust through Research Project Grant RPG-2012-758 (KF and SL), from the National Natural Science Foundation of China Grant Nos. 61137005 and 61520106013 (WC and WP), the project support (ANR-11IDEX-003-02) from the ANR French National Research Agency as well as MS IDI 2012 thesis funding by the IDEX Paris-Saclay, LN2 is an international joint research laboratory, Unité Mixte Internationale UMI 3463, managed by CNRS, Université de Sherbrooke, UdeS, as well as Université de Lyon, UdL, especially including Ecole Centrale de Lyon, INSA Lyon et CPE Lyon, and Université Grenoble-Alpes, UGA. 


\section{Associated content}

Supporting Information

The Supporting Information is available on the web at DOI: $10.1021 / \mathrm{XXX}$

Figures on the dispersion curves in transmission measurements

\section{References}

1. M. Couture, S. S. Zhao and J. F. Masson, Phys. Chem. Chem. Phys., 2013, 15, $11190-11216$.

2. X. D. Hoa, A. G. Kirk and M. Tabrizian, Biosens. Bioelectron., 2007, 23, 151-160.

3. J. Homola, Chem Rev, 2008, 108, 462-493.

4. M. C. Estevez, M. A. Otte, B. Sepulveda and L. M. Lechuga, Anal. Chim. Acta., 2014, 806, 55-73.

5. J.-F. Masson, ACS Sensors, 2017, 2, 16-30.

6. S. S. Aćimović, M. A. Ortega, V. Sanz, J. Berthelot, J. L. Garcia-Cordero, J. Renger, S. J. Maerkl, M. P. Kreuzer and R. Quidant, Nano Letters, 2014, 14, 26362641.

7. P. Chen, M. T. Chung, W. McHugh, R. Nidetz, Y. Li, J. Fu, T. T. Cornell, T. P. Shanley and K. Kurabayashi, ACS Nano, 2015, 9, 4173-4181.

8. H. Im, H. Shao, Y. I. Park, V. M. Peterson, C. M. Castro, R. Weissleder and H. Lee, Nat Biotech, 2014, 32, 490-495.

9. S. H. Lee, N. C. Lindquist, N. J. Wittenberg, L. R. Jordan and S.-H. Oh, Lab on a Chip, 2012, 12, 3882-3890.

10. J. B. Edel, A. A. Kornyshev, A. R. Kucernak and M. Urbakh, Chemical Society Reviews, 2016, 45, 1581-1596.

11. S. W. Zeng, D. Baillargeat, H. P. Ho and K. T. Yong, Chemical Society Reviews, 2014, 43, 3426-3452.

12. A. B. Dahlin, N. J. Wittenberg, F. Hook and S. H. Oh, Nanophotonics, 2013, 2, 83101.

13. A. V. Zayats, Smolyaninov, II and A. A. Maradudin, Phys. Rep.-Rev. Sec. Phys. Lett., 2005, 408, 131-314.

14. J. Breault-Turcot and J.-F. Masson, Anal. Bioanal. Chem., 2012, 403, 1477-1484.

15. J. Henzie, J. Lee, M. H. Lee, W. Hasan and T. W. Odom, in Annu. Rev. Phys. Chem., 2009, vol. 60, pp. 147-165.

16. C. L. Haynes and R. P. Van Duyne, J. Phys. Chem. B, 2001, 105, 5599-5611.

17. J.-F. Masson, M.-P. Murray-Methot and L. S. Live, Analyst, 2010, 135, 1483-1489.

18. R. A. Alvarez-Puebla, A. Agarwal, P. Manna, B. P. Khanal, P. Aldeanueva-Potel, E. Carbo-Argibay, N. Pazos-Perez, L. Vigderman, E. R. Zubarev, N. A. Kotov and L. M. Liz-Marzan, Proceedings of the National Academy of Sciences of the United States of America, 2011, 108, 8157-8161.

19. M. Li, S. K. Cushing and N. Q. Wu, Analyst, 2015, 140, 386-406. 
20. O. Tokel, F. Inci and U. Demirci, Chemical Reviews, 2014, 114, 5728-5752.

21. A. Lopez Gerardo, M. C. Estevez, M. Soler and M. Lechuga Laura, Journal, 2016, 6, 123.

22. W. Zhang, S. Guo, W. S. Pereira Carvalho, Y. Jiang and M. J. Serpe, Analytical Methods, 2016, 8, 7847-7867.

23. W. B. Li, L. Zhang, J. H. Zhou and H. K. Wu, J. Mater. Chem. C, 2015, 3, 64796492.

24. P. L. Stiles, J. A. Dieringer, N. C. Shah and R. R. Van Duyne, in Annual Review of Analytical Chemistry, Annual Reviews, Palo Alto, 2008, vol. 1, pp. 601-626.

25. M. Grzelczak, J. Perez-Juste, P. Mulvaney and L. M. Liz-Marzan, Chem. Soc. Rev., 2008, 37, 1783-1791.

26. M. E. Stewart, C. R. Anderton, L. B. Thompson, J. Maria, S. K. Gray, J. A. Rogers and R. G. Nuzzo, Chem. Rev., 2008, 108, 494-521.

27. A. R. Tao, S. Habas and P. Yang, Small, 2008, 4, 310-325.

28. J. Cao, T. Sun and K. T. V. Grattan, Sensors Actuators B: Chem., 2014, 195, 332351.

29. S. Underwood and P. Mulvaney, Langmuir, 1994, 10, 3427-3430.

30. H. Chen, X. Kou, Z. Yang, W. Ni and J. Wang, Langmuir, 2008, 24, 5233-5237.

31. C. L. Nehl, H. Liao and J. H. Hafner, Nano Lett., 2006, 6, 683-688.

32. A. J. Haes and R. P. Van Duyne, J. Am. Chem. Soc., 2002, 124, 10596-10604.

33. K. Lodewijks, W. Van Roy, G. Borghs, L. Lagae and P. Van Dorpe, Nano Lett., 2012, 12, 1655-1659.

34. R. Bukasov and J. S. Shumaker-Parry, Nano Lett., 2007, 7, 1113-1118.

35. C. Yun-Chorng, C. Hsin-Chan, L. Sih-Chen and G. Tzung-Fang, Nanotechnology, 2013, 24, 095302.

36. P. Hanarp, M. Käll and D. S. Sutherland, The Journal of Physical Chemistry B, 2003, 107, 5768-5772.

37. Y. C. Chang, H. C. Chung, S. C. Lu and T. F. Guo, Nanotechnology, 2013, 24.

38. V. Hafele, A. Trugler, U. Hohenester, A. Hohenau, A. Leitner and J. R. Krenn, Opt. Express, 2015, 23, 10293-10300.

39. S. W. Lee, K. S. Lee, J. Ahn, J. J. Lee, M. G. Kim and Y. B. Shin, Acs Nano, 2011, 5, 897-904.

40. F. Mazzotta, T. W. Johnson, A. B. Dahlin, J. Shaver, S. H. Oh and F. Hook, Acs Photonics, 2015, 2, 256-262.

41. M. A. Otte, M. C. Estevez, L. G. Carrascosa, A. B. Gonzalez-Guerrero, L. M. Lechuga and B. Sepulveda, Journal of Physical Chemistry C, 2011, 115, 53445351.

42. M. Bahramipanah, S. Dutta-Gupta, B. Abasahl and O. J. F. Martin, ACS Nano, 2015, 9, 7621-7633.

43. K.-L. Lee, J.-B. Huang, J.-W. Chang, S.-H. Wu and P.-K. Wei, Sci. Rep., 2015, 5, 8547.

44. T. A. Kelf, Y. Sugawara, R. M. Cole, J. J. Baumberg, M. E. Abdelsalam, S. Cintra, S. Mahajan, A. E. Russell and P. N. Bartlett, Physical Review B, 2006, 74.

45. J. F. Masson, M. P. Murray-Methot and L. S. Live, Analyst, 2010, 135, 1483-1489.

46. H.-S. Leong, J. Guo, R. G. Lindquist and Q. H. Liu, J. Appl. Phys., 2009, 106, 124314. 
47. H. Jiang, T. J. Li, E. Ertorer, J. Yang, J. Sabarinathan and S. Mittler, Sens. Actuator A-Phys., 2013, 189, 474-480.

48. E. H. Lin, M. Y. Pan, M. C. Lee and P. K. Wei, AIP Adv., 2014, 4, 6.

49. M. Couture, K. K. Ray, H.-P. Poirier-Richard, A. Crofton and J.-F. Masson, ACS Sensors, 2016, 1, 287-294.

50. K. C. Vernon, A. M. Funston, C. Novo, D. E. Gomez, P. Mulvaney and T. J. Davis, Nano Lett, 2010, 10, 2080-2086.

51. M. Sarkar, J.-F. Bryche, J. Moreau, M. Besbes, G. Barbillon, B. Bartenlian and M. Canva, Opt Express, 2015, 23, 27376-27390.

52. R. Gillibert, M. Sarkar, J. F. Bryche, R. Yasukuni, J. Moreau, M. Besbes, G. Barbillon, B. Bartenlian, M. Canva and M. L. de la Chapelle, Nanotechnology, 2016, 27, 8.

53. T. H. Reilly, R. C. Tenent, T. M. Barnes, K. L. Rowlen and J. van de Lagemaat, ACS Nano, 2010, 4, 615-624.

54. J. N. Anker, W. P. Hall, O. Lyandres, N. C. Shah, J. Zhao and R. P. Van Duyne, Nat. Mater., 2008, 7, 442-453.

55. L. Pang, G. M. Hwang, B. Slutsky and Y. Fainman, Appl. Phys. Lett., 2007, 91.

56. M. A. Otte, M. C. Estévez, L. G. Carrascosa, A. B. González-Guerrero, L. M. Lechuga and B. Sepúlveda, The Journal of Physical Chemistry C, 2011, 115, 53445351.

57. C. Huang, K. Bonroy, G. Reekman, K. Verstreken, L. Lagae and G. Borghs, Microelectronic Engineering, 2009, 86, 2437-2441.

58. B. Špačková, P. Wrobel, M. Bocková and J. Homola, Proceedings of the IEEE, 2016, 104, 2380-2408.

59. P. Offermans, M. C. Schaafsma, S. R. K. Rodriguez, Y. Zhang, M. Crego-Calama, S. H. Brongersma and J. G. Rivas, ACS Nano, 2011, 5, 5151-5157.

60. J. Ye and P. Van Dorpe, Plasmonics, 2011, 6, 665-671.

61. J. Liu, B. Xu, J. Zhang and G. Song, Plasmonics, 2013, 8, 995-1001.

62. J. B. Lassiter, H. Sobhani, J. A. Fan, J. Kundu, F. Capasso, P. Nordlander and N. J. Halas, Nano Lett., 2010, 10, 3184-3189.

63. Y. Shen, T. Liu, Q. Zhu, J. Wang and C. Jin, Plasmonics, 2015, 10, 1489-1497.

64. G. Lan, S. Liu, Y. Ma, X. Zhang, Y. Wang and Y. Song, Opt. Commun., 2015, 352, 49-54.

65. S. Chen, L. Meng, J. Hu and Z. Yang, Plasmonics, 2015, 10, 71-76.

66. K.-L. Lee, P.-W. Chen, S.-H. Wu, J.-B. Huang, S.-Y. Yang and P.-K. Wei, ACS Nano, 2012, 6, 2931-2939.

67. L. Li, Y. Liang, M. Lu and W. Peng, Plasmonics, 2016, 11, 139-149.

68. A. A. Yanik, A. E. Cetin, M. Huang, A. Artar, S. H. Mousavi, A. Khanikaev, J. H. Connor, G. Shvets and H. Altug, Proc. Natl. Acad. Sci. U. S. A., 2011, 108, 1178411789.

69. Y. Shen, J. Zhou, T. Liu, Y. Tao, R. Jiang, M. Liu, G. Xiao, J. Zhu, Z.-K. Zhou, X. Wang, C. Jin and J. Wang, Nat. Commun., 2013, 4.

70. J. Junesch, T. Sannomiya and A. B. Dahlin, ACS Nano, 2012, 6, 10405-10415.

71. O. R. Bolduc, J. N. Pelletier and J. F. Masson, Anal. Chem., 2010, 82, 3699-3706. 
72. O. R. Bolduc, P. Lambert-Lanteigne, D. Y. Colin, S. S. Zhao, C. Proulx, D. Boeglin, W. D. Lubell, J. N. Pelletier, J. Fethiere, H. Ong and J.-F. Masson, Analyst, 2011, 136, 3142-3148.

73. M. Couture, Y. Z. Liang, H. P. P. Richard, R. Faid, W. Peng and J. F. Masson, Nanoscale, 2013, 5, 12399-12408.

74. M. Couture, L. S. Live, A. Dhawan and J. F. Masson, Analyst, 2012, 137, 41624170.

75. C. A. Saravis, M. S. Wool and H. A. Selenkow, Vox Sang., 1967, 13, 349-353.

76. J. F. Bryche, R. Gillibert, G. Barbillon, M. Sarkar, A. L. Coutrot, F. Hamouda, A. Aassime, J. Moreau, M. L. de la Chapelle, B. Bartenlian and M. Canva, Journal of Materials Science, 2015, 50, 6601-6607.

77. P. P. Zhang, S. M. Yang, L. S. Wang, J. Zhao, Z. C. Zhu, B. Liu, J. Zhong and X. H. Sun, Nanotechnology, 2014, 25, 8.

78. M. Shioi, H. Jans, K. Lodewijks, P. Van Dorpe, L. Lagae and T. Kawamura, Appl. Phys. Lett., 2014, 104, 4.

79. K. C. Bantz, A. F. Meyer, N. J. Wittenberg, H. Im, O. Kurtulus, S. H. Lee, N. C. Lindquist, S. H. Oh and C. L. Haynes, Phys. Chem. Chem. Phys., 2011, 13, 1155111567.

80. J.-F. Bryche, A. Tsigara, B. Bélier, M. L. de la Chapelle, M. Canva, B. Bartenlian and G. Barbillon, Sensors Actuators B: Chem., 2016, 228, 31-35.

81. E. C. Le Ru, E. Blackie, M. Meyer and P. G. Etchegoin, J. Phys. Chem. C, 2007, 111, 13794-13803.

82. D. Cialla, A. Maerz, R. Boehme, F. Theil, K. Weber, M. Schmitt and J. Popp, Anal. Bioanal. Chem., 2012, 403, 27-54.

83. M. Tabatabaei, M. Najiminaini, K. Davieau, B. Kaminska, M. R. Singh, J. J. L. Carson and F. Lagugné-Labarthet, ACS Photonics, 2015, 2, 752-759.

84. W.-D. Li, F. Ding, J. Hu and S. Y. Chou, Opt. Express, 2011, 19, 3925-3936.

85. J. Breault-Turcot, H. P. Poirier-Richard, M. Couture, D. Pelechacz and J. F. Masson, Lab Chip, 2015, 15, 4433-4440.

86. S. Saverot, X. Geng, W. Leng, P. J. Vikesland, T. Z. Grove and L. R. Bickford, RSC Advances, 2016, 6, 29669-29673.

87. S. Nair, C. Escobedo and R. G. Sabat, ACS Sensors, 2017, 2, 379-385.

88. J. Henzie, M. H. Lee and T. W. Odom, Nat Nano, 2007, 2, 549-554.

89. H. Im, S. H. Lee, N. J. Wittenberg, T. W. Johnson, N. C. Lindquist, P. Nagpal, D. J. Norris and S.-H. Oh, ACS Nano, 2011, 5, 6244-6253.

90. A. G. Brolo, E. Arctander, R. Gordon, B. Leathem and K. L. Kavanagh, Nano Letters, 2004, 4, 2015-2018.

91. A. A. Yanik, A. E. Cetin, M. Huang, A. Artar, S. H. Mousavi, A. Khanikaev, J. H. Connor, G. Shvets and H. Altug, Proceedings of the National Academy of Sciences, 2011, 108, 11784-11789. 


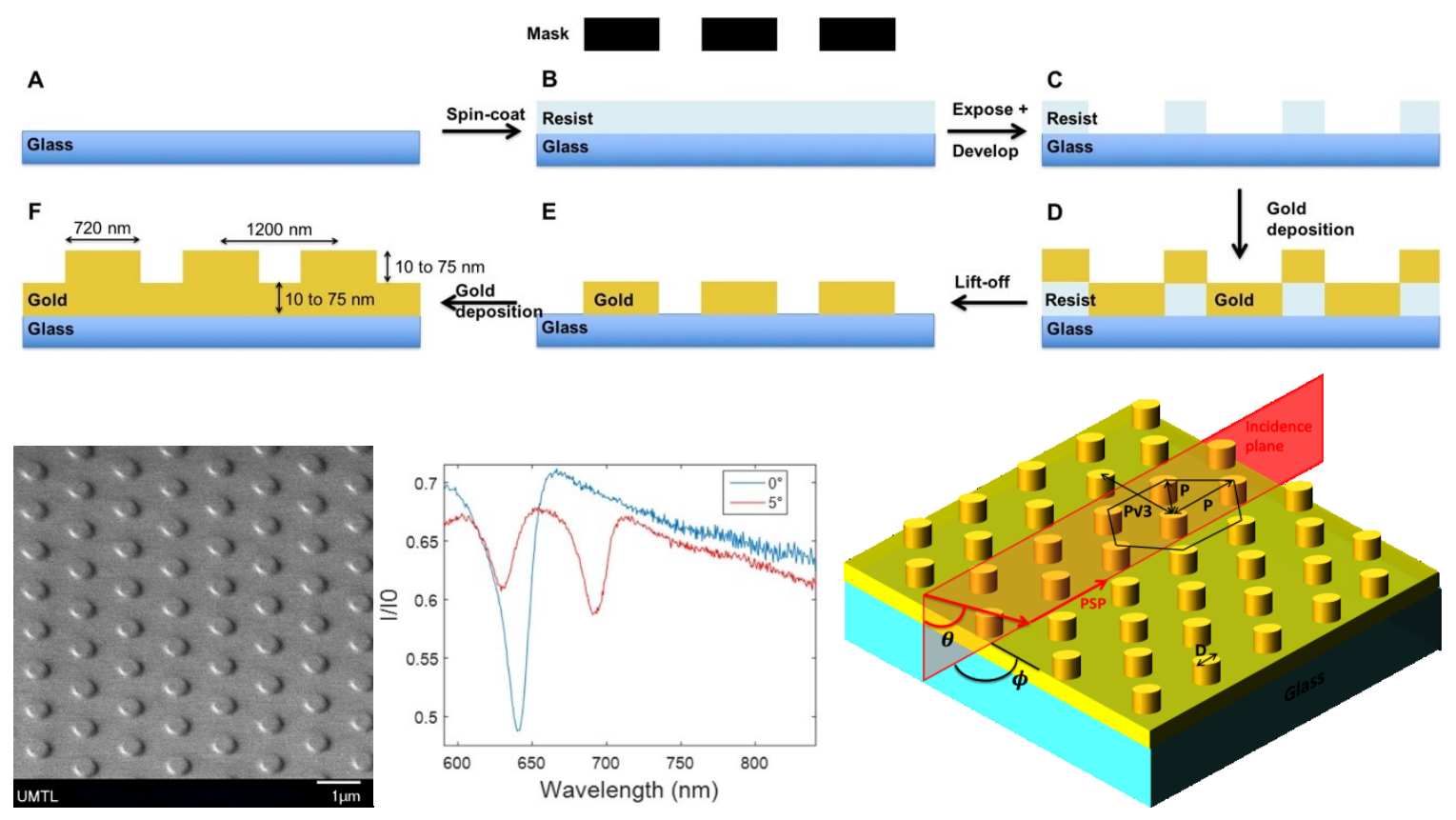

Figure 1. Top) Representation of manufacture process for gold-coated nanodisks; A positive photoresist layer was spin-coated on a 4" glass wafer (A), then UV-light cured through a mask and developed to create an array of holes in the exposed resist (B), followed by the deposition of a gold layer (C), and liftoff of the resist (D) to create an array of nanodisks (E), and finally, the deposition of a gold overlayer to yield the goldcoated nanodisk arrays (F). Bottom left) SEM image of a gold-coated nanodisk arrays with a periodicity of $1200 \mathrm{~nm}$ and a disk diameter of $720 \mathrm{~nm}$. Bottom center) Plasmonic response of nanodisk arrays (ND75film75 - definition in the text) excited in transmission in air for incident angles of $0^{\circ}$ (blue curve) and $5^{\circ}$ (red curve). Bottom right) Representation of the incidence angle $(\theta)$ and rotation angle $(\phi)$. 

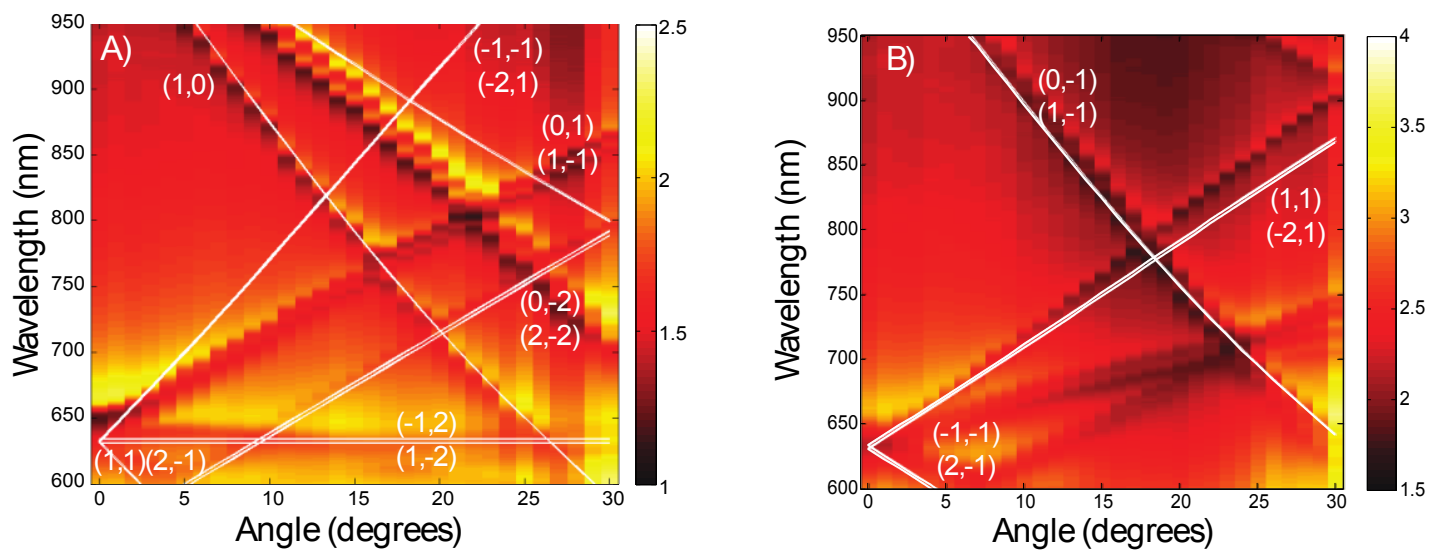

Figure 2. Plasmonic dispersion curves for different orders of BM for a gold nanodisk arrays (ND75film75) with a periodicity of $1200 \mathrm{~nm}$. The calculated modes (white lines) were for the metal/air interface. The color map represents the relative transmission intensity of the gold-coated nanodisk arrays using a smooth $\mathrm{Au}$ film of an equivalent thickness as a reference. The gold-coated nanodisk arrays were aligned at A) $0^{\circ}$ rotation angle (incident light oriented with the $(1,0)$ mode) and B) $90^{\circ}$ rotation angle (perpendicular to the $(1,0)$ mode). Other modes can also be seen on the plasmon dispersion curve, which can be associated to the BM of the plasmon at the glass-metal interface. 

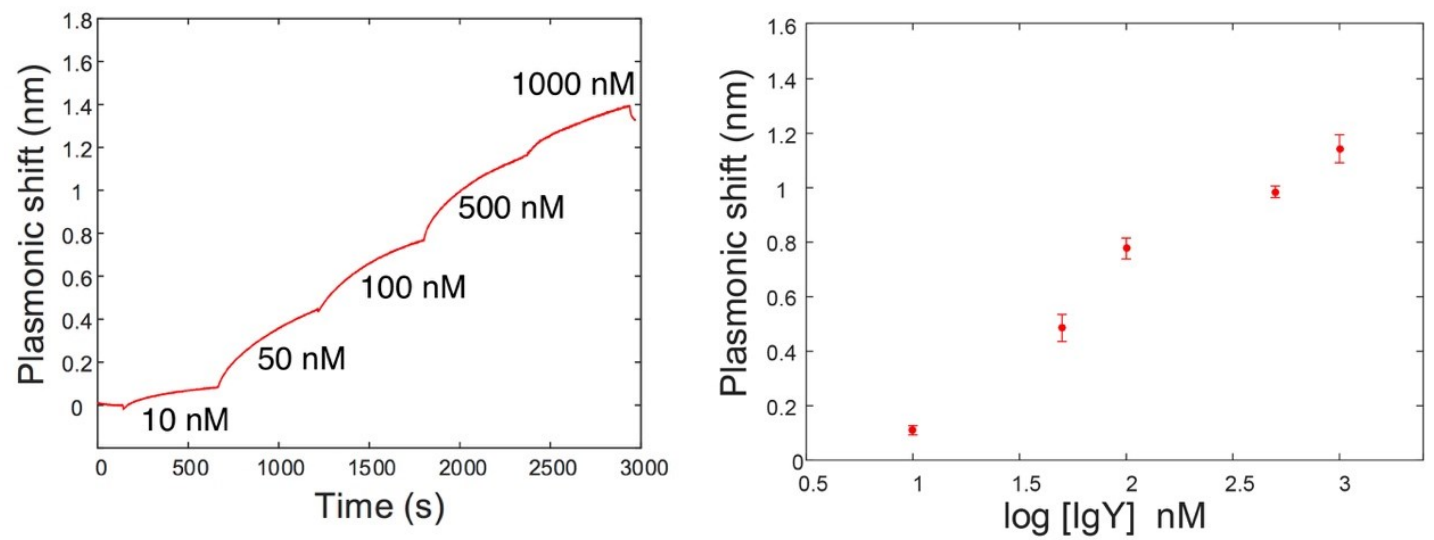

Figure 3. Left) Sensorgrams for the detection of IgY with the gold-coated nanodisk arrays. Concentrations of 10, 50, 100, 500 and $1000 \mathrm{nM}$ were successively injected in a fluidic cell. Right) IgY calibration curves for structures of gold-coated nanodisk arrays excited in transmission using a fluidic cell. 

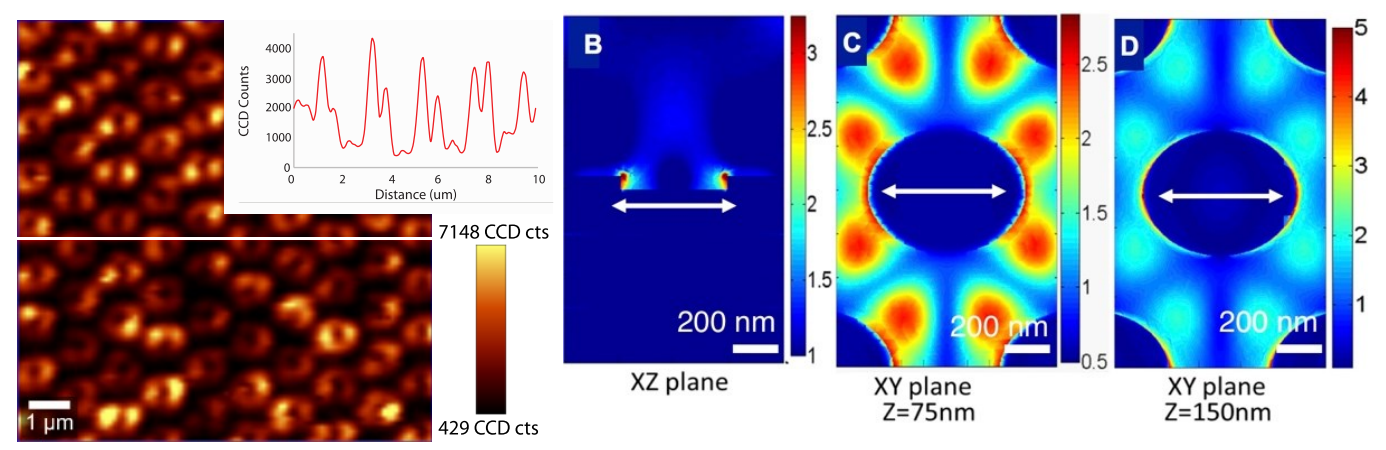

Figure 4. A) Raman imaging of a gold-coated nanodisk arrays (ND75film75) with 4nitrobenzenethiol (4-NBT). The image was acquired with a $633 \mathrm{~nm}$ laser and $100 \mathrm{~nm}$ steps between pixels and $100 \mathrm{~ms}$ integration time per pixel. The image represents a false color Raman map of the intensity of 4-NBT peak at $1335 \mathrm{~cm}^{-1}$ for a scanning area of 10 $\mu \mathrm{m} \times 10 \mu \mathrm{m}$. The inset shows a cross-section of the Raman intensity corresponding to the white line in the SERS image. B-D) The field distribution at resonance wavelength (644 $\mathrm{nm}$ ) was calculated in 3D under polarized light for the gold-coated nanodisk arrays. The light was impinging from the top of the image $\mathrm{B}$, directly on the nanodisk array-air interface, similar as to the Raman imaging experiment. The field was normalized to the incident $1 \mathrm{~V} / \mathrm{m}$. The arrow represents the direction of the polarization. The region of highest field intensity was located at the top rim of the nanodisk. The field distribution at resonance wavelength $(644 \mathrm{~nm})$ was calculated in the X-Y plane under polarized light for the glass-gold interface for the gold film-nanodisk interface $(Z=75 \mathrm{~nm}, C)$ and for the nanodisk-air interface $(Z=150 \mathrm{~nm}, \mathrm{D})$. The images also represent normalized false color maps of the field distribution and the arrows represent the orientation of the polarization of the incident light. 
Table 1. Plasmonic sensing properties of gold-coated nanodisk arrays.

$\begin{array}{lllll} & \text { ND50film50 } & \text { ND50film75 } & \text { ND75film50 } & \text { ND75film75 } \\ \text { Sensitivity (nm/RIU) } & 583 \pm 1 & 601 \pm 2 & 599 \pm 2 & 599 \pm 3 \\ \text { FWHM (nm) } & 14 \pm 2 & 14 \pm 4 & 13 \pm 1 & 14 \pm 1 \\ \text { FOM } & 42 & 43 & 46 & 43 \\ \text { RI resolution (RIU) } & 1.9 \times 10^{-5} & 1.8 \times 10^{-5} & 1.8 \times 10^{-5} & 1.9 \times 10^{-5} \\ \Delta \lambda_{\text {SPR }}(\mathrm{nm}) \text { IgY } & 0.5 \pm 0.1 & 0.4 \pm 0.1 & 0.2 \pm 0.1 & 0.5 \pm 0.1 \\ \Delta \lambda_{\text {SPR }}(\mathrm{nm}) \text { anti-IgY } & 2.3 \pm 0.2 & 2.6 \pm 0.2 & 1.9 \pm 0.2 & 2.8 \pm 0.2\end{array}$

Table 2. Comparison of analytical parameters of 2D plasmonic structures

\begin{tabular}{|c|c|c|c|c|c|c|c|c|}
\hline Structure & $\begin{array}{c}\text { Sensitivity } \\
\text { (nm/RIU) }\end{array}$ & $\begin{array}{l}\text { FWHM } \\
(\mathrm{nm})\end{array}$ & $\begin{array}{c}\text { FOM } \\
\left(\mathrm{RIU}^{-1}\right)\end{array}$ & $\begin{array}{l}\text { Resolution } \\
\text { (RIU) }\end{array}$ & $\begin{array}{l}\text { SERS } \\
\text { EF }\end{array}$ & $\begin{array}{l}\text { Features } \\
(\mathrm{nm})\end{array}$ & $\begin{array}{l}\text { Periodicity } \\
\text { (nm) }\end{array}$ & Reference \\
\hline Crossed gratings & 647 & 50 & 14 & $10^{-5}$ & NA & NA & 550 & 87 \\
\hline Nanodisk arrays & $161^{\mathrm{a}}$ & $10.5^{\mathrm{a}}$ & $15^{\mathrm{a}}$ & $2 \times 10^{-5 b}$ & $\begin{array}{l}2.6 \mathrm{x} \\
10^{7 \mathrm{c}}\end{array}$ & 100 & 500 & a $37 ;$ b 56 ; 76 \\
\hline Nanohole arrays & $313^{\mathrm{d}}$ & $14.5^{\mathrm{d}}$ & $23^{\mathrm{d}}$ & $2 \times 10^{-5} \mathrm{e}$ & $10^{5 \mathrm{f}}$ & 100 & 400 & d $88 ;$ e $89 ;$ f 90 \\
\hline Nanomushrooms & 1015 & 9.5 & 108 & $2 \times 10^{-4}$ & NA & 285 & 610 & 69 \\
\hline $\begin{array}{l}\text { Suspended } \\
\text { nanohole arrays } \\
\text { (Fano) }\end{array}$ & 717 & 4 & 162 & NA & NA & 230 & 580 & 91 \\
\hline $\begin{array}{l}\text { Gold-coated } \\
\text { nanodisk arrays }\end{array}$ & 599 & 14 & 43 & $1.9 \times 10^{-5}$ & $10^{7}$ & 720 & 1200 & this work \\
\hline
\end{tabular}


TOC Graph:

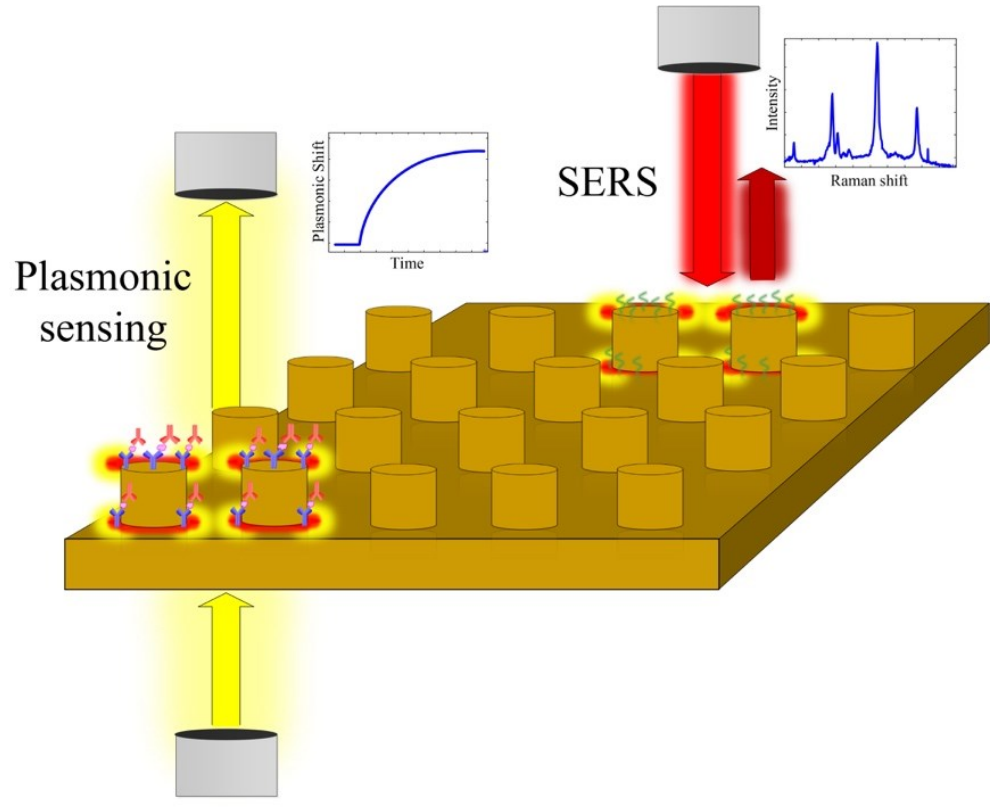

